\title{
Triple Orthogonal Labeling of Glycans by Applying Photoclick Chemistry
}

\author{
Verena F. Schart, Jessica Hassenrück, Anne-Katrin Späte, Jeremias E. G. A. Dold, \\ Raphael Fahrner, and Valentin Wittmann*[a]
}

\begin{abstract}
Bioorthogonal labeling of multiple biomolecules is of current interest in chemical biology. Metabolic glycoengineering (MGE) has been shown to be an appropriate approach to visualizing carbohydrates. Here, we report that the nitrile imine-alkene cycloaddition (photoclick reaction) is a suitable ligation reaction in MGE. Using a mannosamine derivative with an acrylamide reporter group that is efficiently metabolized by cells and that quickly reacts in the photoclick reaction, we labeled sialic acids on the surface of living cells. Screening of several alkenes showed that a previously reported carbamate-linked methylcyclopropene reporter that is well suited for the inverse-electrondemand Diels-Alder $\left(\mathrm{DA}_{\text {inv }}\right)$ reaction has a surprisingly low reactivity in the photoclick reaction. Thus, for the first time, we were able to triply label glycans by a combination of $\mathrm{DA}_{\text {inv }}$ photoclick, and copper-free click chemistry.
\end{abstract}

Bioorthogonal ligations have become an important tool in the field of chemical biology. ${ }^{[1]}$ In particular, azide-alkyne cycloaddition ${ }^{[2]}$ and the inverse-electron-demand Diels-Alder $\left(\mathrm{DA}_{\mathrm{inv}}\right)$ reaction ${ }^{[3]}$ have been intensively studied and employed for a wide range of applications. During the last ten years, another ligation reaction, the nitrile imine-alkene cycloaddition (photoclick reaction) has gained attention in bioconjugation. ${ }^{[4]}$ In this reaction, UV irradiation of a tetrazole forms a nitrile imine in situ that rapidly reacts with an alkene (Scheme $1 \mathrm{~A})$. The advantages of the photoclick reaction are a high reaction rate, the formation of a fluorescent product, and the possibility to control the reaction in a spatial and temporal manner by selective irradiation. So far, the reaction has been successfully applied to label proteins, ${ }^{[4 \mathrm{~b}-\mathrm{e}, 5]} \mathrm{DNA}^{[6]}$ and $\mathrm{RNA}_{1}{ }^{[7]}$ although glycans, another class of essential biomolecules, have not yet been labeled.

An important technique to image cellular carbohydrates is metabolic glycoengineering (MGE) ${ }^{[8]}$ In this approach, an unnatural monosaccharide derivatized with a chemical reporter group is metabolized by cells or organisms and incorporated into glycoconjugates. The unnatural reporter group can then

[a] Dr. V. F. Schart, J. Hassenrück, Dr. A.-K. Späte, J. E. G. A. Dold, R. Fahrner, Prof. Dr. V. Wittmann

Department of Chemistry and Konstanz Research School Chemical Biology (KoRS-CB)

University of Konstanz

78457 Konstanz (Germany)

E-mail:mail@valentin-wittmann.de

A)

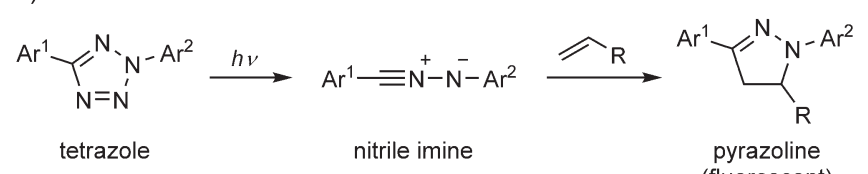

B)

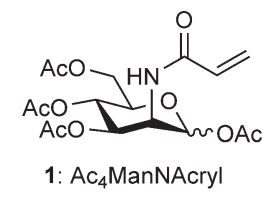

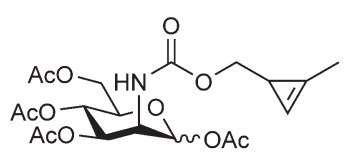

2: $\mathrm{Ac}_{4} \mathrm{ManNCyoc}$

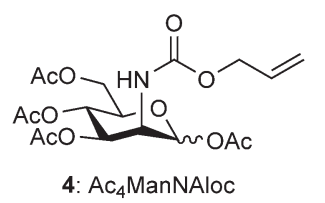

Scheme 1. A) Photoclick reaction of tetrazoles. B) $A c_{4}$ ManNAcryl and additional mannosamine derivatives for MGE.

be reacted in a bioorthogonal ligation to visualize or isolate the glycoconjugate. High labeling efficiency is achieved with carbohydrate derivatives that both are well accepted by the enzymes involved in glycan biosynthesis and that undergo a fast labeling reaction. With the $\mathrm{DA}_{\text {inv }}$ reaction for labeling, however, both criteria are often not met at the same time. For example, we could show that alkene-modified ManNAc derivatives with shorter spacers between the alkene (acting as the dienophile) and the electron-withdrawing carbonyl group are better accepted by the enzymes but react more slowly in the $D_{\text {inv }}$ reaction with tetrazines than do derivatives with longer chain lengths. ${ }^{[9]}$ The lower $D A_{\text {inv }}$ reactivity of the electron-poor, short-chain derivatives is in line with a reaction control by a

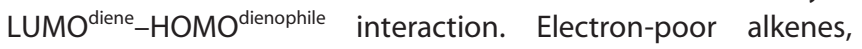
however, are the preferred substrates for the photoclick reaction, ${ }^{[4 d, 10]}$ and the small acrylamide group reacts especially fast in this ligation. ${ }^{[4 d, 5 b]}$ This prompted us to investigate the photoclick reaction as an interesting expansion of the MGE toolbox.

Multiple-color imaging by using bioorthogonal ligations that are orthogonal to each other is of current interest in biomolecular chemistry. ${ }^{[1,11]}$ The combination of click and DA $A_{\text {inv }}$ chemistry, for example, has been used many times, including in MGE, 
to achieve dual labeling. ${ }^{[12]}$ However, examples in which triple labeling with three orthogonal reactions has been achieved are rare $^{[13]}$ and have not yet been reported in MGE. Prescher and co-workers investigated a combination of photoclick and $D A_{\text {inv }}$ reactions to label two species. ${ }^{[12 d]}$ They could show that 3,3-disubstituted cyclopropenes react readily with nitrile imines but not with tetrazines. In contrast, 1,3-disubstituted derivatives react readily with both. An example of an alkene substrate that readily undergoes an $D A_{\text {inv }}$ reaction but does not react in the photoclick reaction has not yet been reported.

Herein we show that the photoclick reaction is a suitable ligation for MGE. Acrylamide-functionalized mannosamine derivative 1 (Scheme 1B) is efficiently metabolized by cells due to its structural similarity to natural ManNAc and it quickly reacts with nitrile imines to allow the visualization of sialic acids on the surface of living cells. Furthermore, we found that the previously reported ${ }^{[12 e, 14]} \mathrm{Ac}_{4}$ ManNCyoc (2), which is known to react readily with tetrazines, has a surprisingly low reactivity in the photoclick reaction. This enabled us to triply label glycans by combining $\mathrm{DA}_{\text {inv }}$ photoclick, and click chemistry.

For the synthesis of 1 we started from $\mathrm{N}$-Boc-protected peracetylated mannosamine, ${ }^{[15]}$ which was $\mathrm{N}$-deprotected and subsequently treated with acryloyl chloride as shown in the Supporting Information (Scheme S1). To confirm that 1 was accepted by the biosynthetic pathway, we applied it for MGE and analyzed the resulting sialic acids after labeling with 1,2-diamino4,5-methylenedioxybenzene (DMB; Scheme 2, path A). In this technique, the sialic acids are released from metabolically engi-

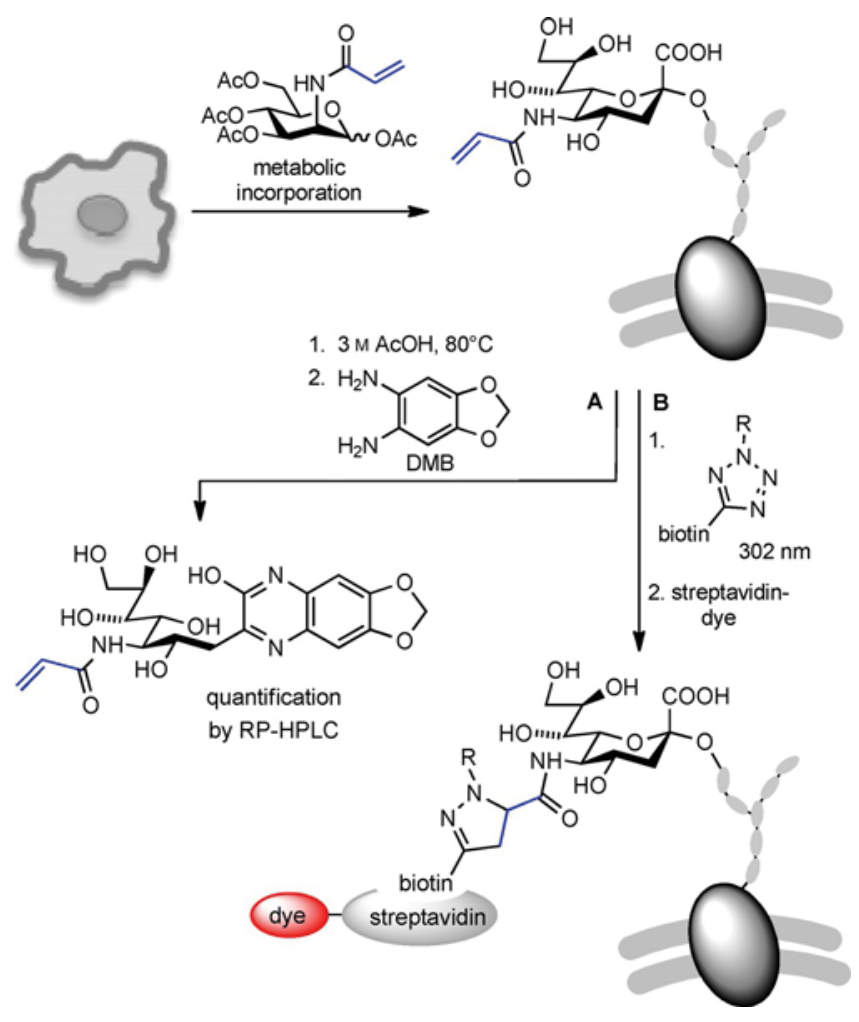

Scheme 2. MGE strategy with DMB labeling to determine the incorporation efficiency (path $A$ ) and the photoclick reaction as ligation for visualization (path B). neered cells by treatment with acetic acid followed by selective labeling of the $\alpha$-keto acids with DMB. ${ }^{[16]}$ The DMB-labeled sialic acids were analyzed by RP-HPLC/MS and correlated to separately synthesized reference compounds (Figure S1). We found that $43 \%$ of all released sialic acids bear the acrylamide functionality (Figures S2 and S3); this demonstrates the high acceptance of this small reporter group by the enzymes. In comparison, mannosamine derivatives modified with a bulky norbornene were found to be incorporated with an efficiency of only $1 \% .{ }^{[17]}$ These findings also show that the acrylamide group is stable during metabolization and not attacked by thiols in a Michael-type addition. This stability in the cellular environment is in line with results of the Liu lab. ${ }^{[4 d, 18]}$ Being stable in a cellular environment and featuring a high incorporation efficiency, $\mathrm{Ac}_{4}$ ManNAcryl (1) is a promising new derivative for MGE.

To be able to apply the photoclick reaction in MGE, we first developed suitable reaction conditions. From the range of tetrazoles ${ }^{[4 c, 19]}$ that have been used for various applications, we chose to use tetrazoles 6 and $7^{[20]}$ (Scheme 3) because they are

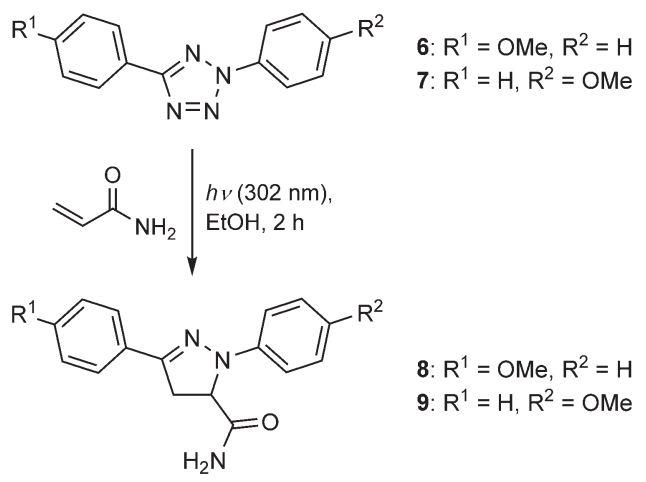

Scheme 3. Photoclick reaction of tetrazoles 6 and 7 to yield pyrazolines 8 and 9.

synthetically easily accessible and were previously successfully used in a biological environment. ${ }^{[5 b, 21]}$ Furthermore, their reaction with acrylamide on a preparative scale has been reported to give high yields of pyrazolines $\mathbf{8}$ and $\mathbf{9}$ upon irradiation at $302 \mathrm{~nm},{ }^{[10 \mathrm{~b}]}$ which we could confirm. The fluorescent pyrazolines have an absorbance maximum at $360 \mathrm{~nm}$ (Figure S4) and fluorescence maxima at 445 (8) and $485 \mathrm{~nm}$ (9), respectively (Figure S5).

For an efficient screening of reaction conditions and of the reactivity of different substrates, we exploited the fluorogenic character of the photoclick reaction and developed a platereader assay in a 96-well format with a fluorescent readout. A solution containing tetrazole $(1 \mathrm{~mm})$ and alkene $(100 \mu \mathrm{M})$ in ethanol was irradiated at $302 \mathrm{~nm}$ with a UV lamp. The relative amount of formed pyrazolines was then quantified by determination of the fluorescence intensity. We first varied the irradiation time between $10 \mathrm{~s}$ and 5 min with 1 as reaction partner (Figure S6) and found that an irradiation of $1 \mathrm{~min}$ is sufficient and also compatible with biological experiments. Next, a suitable solvent needed to be determined because pure ethanol is 
cytotoxic. A well-established solvent for cell experiments is phosphate-buffered saline (PBS), which maintains the osmotic pressure of the cells. PBS, however, has a high content of chloride ions, which are reported to slow down the photoclick reaction. ${ }^{[22]}$ Thus, we investigated the efficiency of the photoclick reaction in water and PBS, and found both solvents to be similarly suitable (Figure S7).

We next applied the plate-reader assay to compare the reactivity of different carbohydrate derivatives in the photoclick reaction. Beside 1 we used 2 and $\mathrm{Ac}_{4}$ ManNNorb (3; for its synthesis, see Scheme S2). Norbornenes ${ }^{[4,23]}$ and several amidelinked methylcyclopropenes ${ }^{[5 b, 12 d, 24]}$ are known to undergo photoclick reaction; however, carbamate-linked methylcyclopropenes, such as $\mathbf{2}$, have not yet been investigated in this context. We also included the terminal alkene $\mathrm{Ac}_{4}$ ManNAloc $(4)^{[9 a]}$ because allyl ethers have been applied for the photoclick reaction although with variable efficiency. ${ }^{[4 d, 7,21]}$

We detected remarkable differences in the reactivities of sugars 1-4 in the photoclick reaction with tetrazole 6 (Figure 1). The highest fluorescence signal was detected for $\mathbf{1}$.

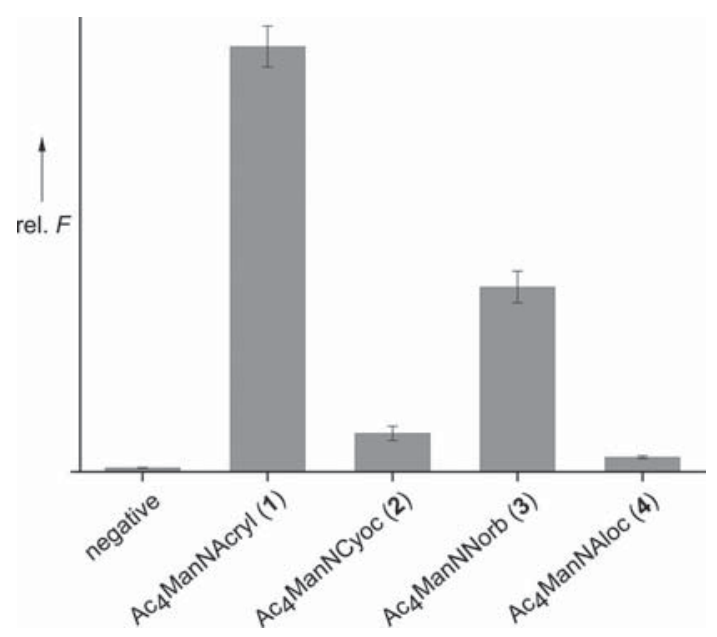

Figure 1. Results of the plate-reader assay with different alkene-modified mannosamines. Solutions containing $1 \mathrm{~mm}$ tetrazole 6 and $100 \mu \mathrm{m}$ carbohydrate in ethanol were irradiated for $1 \mathrm{~min}(302 \mathrm{~nm})$. In the negative control, the carbohydrate was omitted. Fluorescence $F$ was detected at $445 \mathrm{~nm}$ after excitation at $360 \mathrm{~nm}$. Measurements were taken from two independent experiments with two replicates each.

The signal intensity observed with $\mathbf{3}$ was less than half of that, but still well above background. Surprisingly, 2 resulted in only very low intensity, and hardly any signal was detected for 4 . Similar trends were obtained with tetrazole 7 (Figure S8). The unexpectedly low fluorescence intensity obtained with cyclopropene derivative $\mathbf{2}$ is remarkable because all previously tested cyclopropenes reacted efficiently in the photoclick reaction. ${ }^{[5,12 d, 24]}$ This lack of reactivity of $\mathbf{2}$ in the photoclick reaction prompted us to investigate whether it could be exploited to achieve dual or even triple orthogonal labeling of carbohydrates.

Next, we established the application of the photoclick reaction in MGE. Even though the photoclick reaction generates fluorescent pyrazolines, we decided to apply a two-step labeling procedure because this allows the use of dyes that are better suited for fluorescence microscopy in living cells. Thus, we synthesized tetrazole-biotin derivatives 10 and 11<smiles>CCCOCCCNC(=O)CCCC[C@H]1SCC2NC(=O)NC21</smiles>

(Scheme S3) that-after photoclick reaction-can be labeled with streptavidin-AlexaFluor 555 (streptavidin-AF555). Both derivatives have an amino-oligo(ethylene glycol) (OEG) linker to ensure solubility. Their syntheses started from tetrazoles 6 and 7 , respectively, as shown in the Supporting Information.

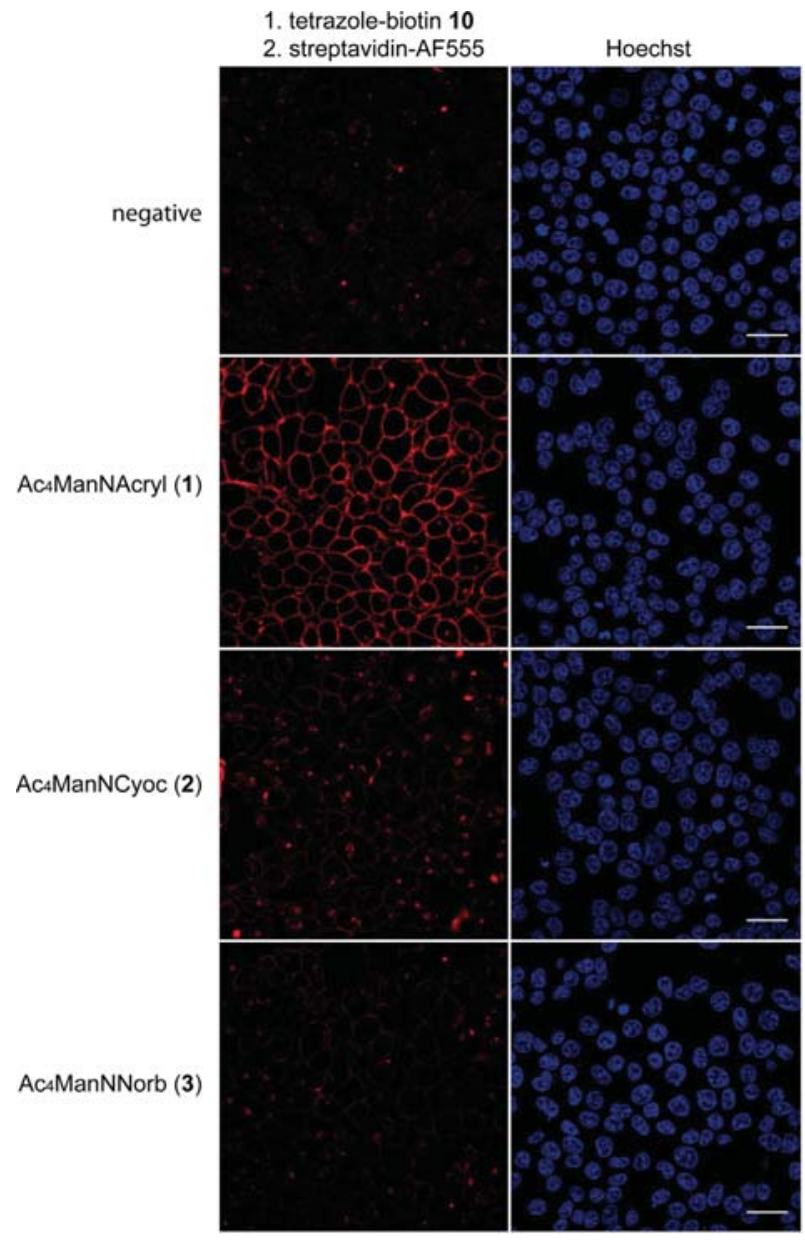

Figure 2. Confocal fluorescence microscopy images of cells labeled by using the photoclick reaction. HEK 293T cells were incubated with $100 \mu \mathrm{M} 1,2$ or 3 for two days. DMSO was added as solvent control. For visualization, cells were treated with 10 ( $1 \mathrm{~min}, 302 \mathrm{~nm}$; $15 \mathrm{~min}, \mathrm{RT}$ ) followed by streptavidinAF555. Nuclei were stained with Hoechst 33342. Scale bars: $30 \mu \mathrm{m}$. 
A)

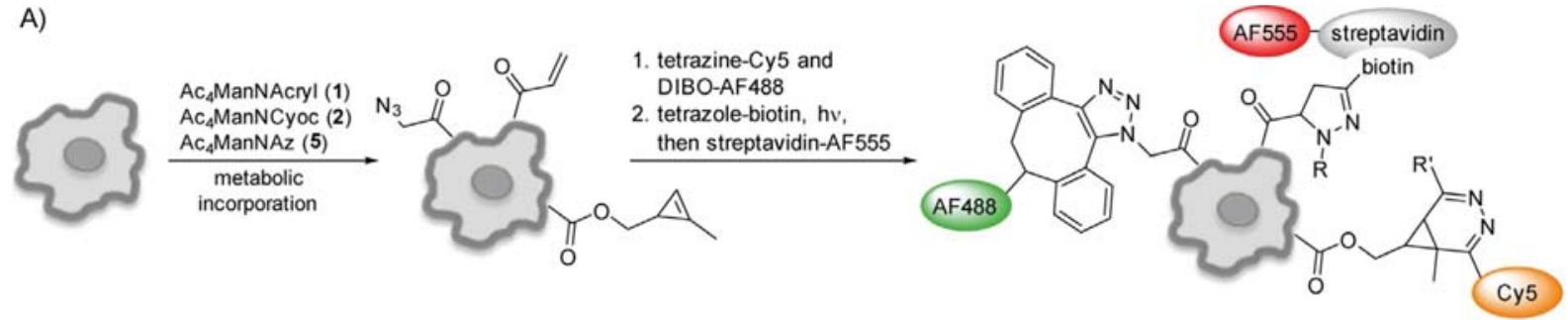

B)

ACAManNAz (5, $10 \mu \mathrm{M})$ AcaManNCyoc $(2,100 \mu \mathrm{M})$ Ac4ManNAcryl (1, $80 \mu \mathrm{M})$

AcaManNCyoc (2, $100 \mu \mathrm{M})$ AcaManNAcryl (1, $80 \mu \mathrm{M})$

AcaManNAz $(5,10 \mu \mathrm{M})$ AcAManNAcryl (1,80 بM)

$\operatorname{Ac} 4 \operatorname{ManNAz}(5,10 \mu \mathrm{M})$ AcaManNCyoc (2,100 $\mu \mathrm{M})$

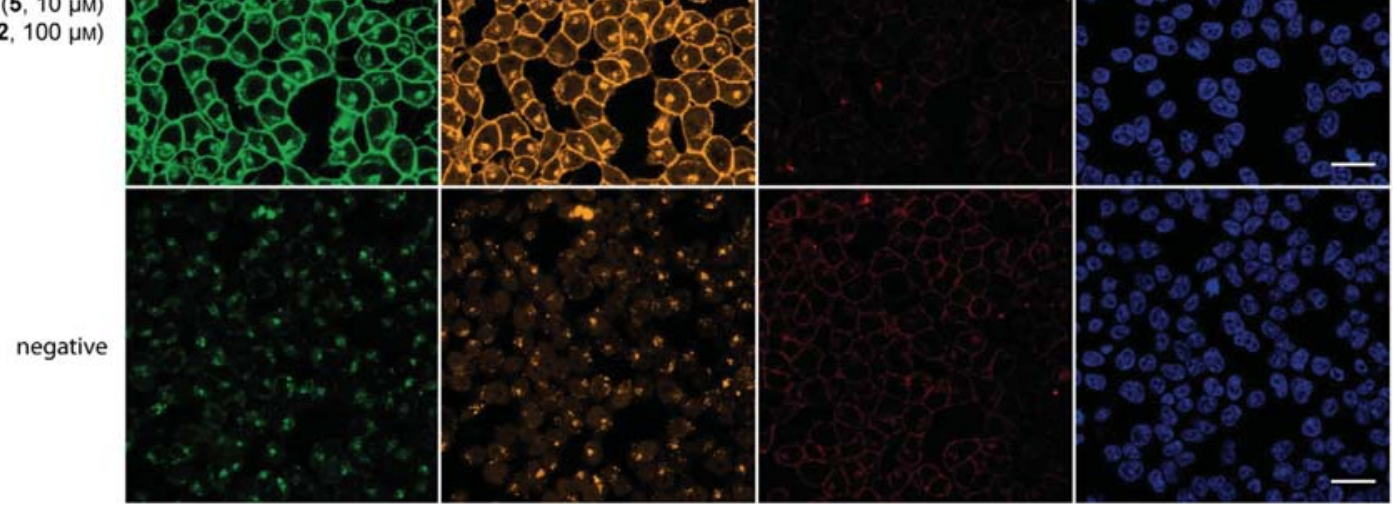

Figure 3. A) Triple labeling strategy combining $\mathrm{DA}_{\text {inv }}$ click, and photoclick chemistry. B) Confocal fluorescence microscopy of triply labeled cells. HEK $293 \mathrm{~T}$ cells were incubated with $5(10 \mu \mathrm{M}), \mathbf{2}(100 \mu \mathrm{M})$, and $1(80 \mu \mathrm{M})$ for $48 \mathrm{~h}$. As controls, only two sugars were added or only DMSO. For labeling, cells were treated with DIBO-AF488 $\left(20 \mu \mathrm{M}, 1 \mathrm{~h}, 37^{\circ} \mathrm{C}\right)$ and tetrazine-Cy5 $\left(40 \mu \mathrm{M}, 1 \mathrm{~h}, 37^{\circ} \mathrm{C}\right)$ followed by the addition of 10 (1 min, $302 \mathrm{~nm}$; $\left.15 \mathrm{~min}, \mathrm{RT}\right)$ and streptavidin-AF555. Nuclei were stained with Hoechst 33342. Scale bars: $30 \mu \mathrm{m}$.

The UV spectra of both derivatives were similar to those of 6 and 7 (Figure S4).
For the MGE experiments we used 1 and 3 because of their high photoclick reactivity and 2 to test whether its low reactiv- 
ity according to the plate-reader assay could be exploited for orthogonal labeling. HEK 293T cells were grown with the respective monosaccharide, treated with $10,{ }^{[25]}$ irradiated for $1 \mathrm{~min}$ by UV light $(302 \mathrm{~nm})$, and then incubated at room temperature for $15 \mathrm{~min}$. The glycoconjugates were visualized by the addition of streptavidin-AF555 (Scheme 2, path B) and imaged by confocal fluorescence microscopy (Figure 2). $\mathrm{Ac}_{4}$ ManNAcryl (1) resulted in a clear membrane staining, whereas neither $\mathrm{Ac}_{4}$ ManNCyoc (2) nor $\mathrm{Ac}_{4}$ ManNNorb (3) showed any significant fluorescence, similar to the negative control. As $\mathbf{3}$ is reasonably reactive in the photoclick reaction, according to the plate reader assay (Figure 1), the lack of membrane staining is likely due to a low incorporation efficiency. Interestingly, although it has recently been reported that the nitrile imines formed during the photoclick reaction can react with a variety of nucleophiles present in a biological system, ${ }^{[26]}$ we observed only low background signal under the conditions used.

Because 2 does not lead to a membrane staining after photoclick reaction and 1 does not react in the $\mathrm{DA}_{\text {inv }}$ reaction (Figure S9), we were able to use the two carbohydrates in an orthogonal dual-labeling strategy (Figure S10). Furthermore, we could show that the photoclick reaction is also orthogonal to strain-promoted azide-alkyne cycloaddition by combining 1 and $\mathrm{Ac}_{4} \mathrm{ManNAz}$ (5) in an MGE experiment (Figure S11). These results set the stage for the exploration of triple orthogonal labeling of glycans by combining all three derivatives 1, 2, and 5 (Figure 3A). Thus, we grew HEK 293T cells with $80 \mu \mathrm{M}$ 1, $100 \mu \mathrm{M}$ 2, and $10 \mu \mathrm{M}$ 5. The different concentrations were chosen to obtain comparable cell-surface staining intensities in all three channels. For triple labeling, we first performed the $\mathrm{DA}_{\mathrm{inv}}$ reaction by using a tetrazine-Cy5 conjugate and the click reaction by using a dibenzocyclooctyne-AlexaFluor 488 conjugate (DIBO-AF488; for formulas see the Supporting Information) simultaneously, followed by the photoclick reaction. As shown in Figure 3B, first row, clear membrane staining was detected in all three channels. Omission of one of the sugars resulted in missing membrane staining in the respective channel (rows 2-4), thus confirming the absence of cross reactivity. When pure DMSO was added (negative control), membrane staining could not be detected in any channel.

In summary, we have demonstrated that the photoclick reaction can be used to visualize the cell-surface carbohydrates of metabolically engineered living cells. $A c_{4}$ ManNAcryl (1), which is modified with the smallest chemical reporter group used in MGE so far, turned out to be metabolically stable enough to be incorporated into sialoglycans with high efficiency and subsequently be labeled with a tetrazole derivative. Labeling by the photoclick reaction is thus a useful expansion of the toolbox of labeling reactions that can be used to visualize carbohydrates. With the possibility of achieving spatiotemporal control by selective irradiation, the reaction opens up new avenues in studying carbohydrate-dependent processes in a space- and time-resolved manner. The surprising observation that $\mathrm{Ac}_{4}$ ManNCyoc (2) has only low reactivity in the photoclick reaction enabled us to achieve triple labeling in MGE for the first time. As the simultaneous visualization of multiple biomole- cules is of general interest in chemical biology, we expect the triple-labeling approach to have many potential applications.

\section{Acknowledgements}

This work was supported by the Deutsche Forschungsgemeinschaft (SPP 1623 and SFB 969, project B05), the University of Konstanz, and the Konstanz Research School Chemical Biology (stipend for R.F.). We thank Felix Englmaier for synthetic support, the Bioimaging Center of the University of Konstanz for providing the fluorescence microscopy instrumentation, and the NMR Core Facility of the University of Konstanz for providing the NMR instrumentation.

\section{Conflict of Interest}

The authors declare no conflict of interest.

Keywords: bioorthogonal reactions - carbohydrates • click chemistry $\cdot$ metabolic engineering $\cdot$ tetrazines

[1] a) J. M. Baskin, C. R. Bertozzi, QSAR Comb. Sci. 2007, 26, 1211-1219; b) E. M. Sletten, C. R. Bertozzi, Angew. Chem. Int. Ed. 2009, 48, 69746998; Angew. Chem. 2009, 121, 7108-7133; c) C. P. Ramil, Q. Lin, Chem. Commun. 2013, 49, 11007-11022.

[2] M. Meldal, C. W. Tornøe, Chem. Rev. 2008, 108, 2952-3015.

[3] a) E. Kozma, O. Demeter, P. Kele, ChemBioChem 2017, 18, 486-501; b) S. Mayer, K. Lang, Synthesis 2017, 49, 830-848.

[4] a) R. Huisgen, M. Seidel, G. Wallbillich, H. Knupfer, Tetrahedron 1962, 17 3-29; b) W. Song, Y. Wang, J. Qu, M. M. Madden, Q. Lin, Angew. Chem. Int. Ed. 2008, 47, 2832-2835; Angew. Chem. 2008, 120, 2874-2877 c) W. Song, Y. Wang, J. Qu, Q. Lin, J. Am. Chem. Soc. 2008, 130, 96549655; d) Y.-J. Lee, B. Wu, J. E. Raymond, Y. Zeng, X. Fang, K. L. Wooley, W. R. Liu, ACS Chem. Biol. 2013, 8, 1664-1670; e) E. Kaya, M. Vrabel, C. Deiml, S. Prill, V. S. Fluxa, T. Carell, Angew. Chem. Int. Ed. 2012, 51, 4466 4469; Angew. Chem. 2012, 124, 4542-4545.

[5] a) J. Wang, W. Zhang, W. Song, Y. Wang, Z. Yu, J. Li, M. Wu, L. Wang, J. Zang, Q. Lin, J. Am. Chem. Soc. 2010, 132, 14812-14818; b) Z. Yu, Y. Pan, Z. Wang, J. Wang, Q. Lin, Angew. Chem. Int. Ed. 2012, 51, $10600-$ 10604; Angew. Chem. 2012, 124, 10752-10756.

[6] S. Arndt, H.-A. Wagenknecht, Angew. Chem. Int. Ed. 2014, 53, $14580-$ 14582; Angew. Chem. 2014, 126, 14808-14811.

[7] J. M. Holstein, D. Stummer, A. Rentmeister, Chem. Sci. 2015, 6, 1362 1369.

[8] a) P. R. Wratil, R. Horstkorte, W. Reutter, Angew. Chem. Int. Ed. 2016, 55, 9482-9512; Angew. Chem. 2016, 128, 9632 -9665; b) K. K. Palaniappan C. R. Bertozzi, Chem. Rev. 2016, 116, 14277-14306.

[9] a) A.-K. Späte, V. F. Schart, S. Schöllkopf, A. Niederwieser, V. Wittmann, Chem. Eur. J. 2014, 20, $16502-16508$; b) J. E. G. A. Dold, J. Pfotzer, A.-K. Späte, V. Wittmann, ChemBioChem 2017, 18, 1242-1250.

[10] a) K. N. Houk, J. Sims, C. R. Watts, L. J. Luskus, J. Am. Chem. Soc. 1973, 95, 7301-7315; b) Y. Wang, C. I. Rivera Vera, Q. Lin, Org. Lett. 2007, 9, $4155-4158$.

[11] a) T. Kurpiers, H. D. Mootz, Angew. Chem. 2009, 121, 1757-1760; b) M. F. Debets, J. C. M. van Hest, F. P. J. T. Rutjes, Org. Biomol. Chem. 2013, 11, 6439-6455.

[12] a) M. R. Karver, R. Weissleder, S. A. Hilderbrand, Angew. Chem. Int. Ed. 2012, 51, 920-922; Angew. Chem. 2012, 124, 944-946; b) J. Schoch, M. Staudt, A. Samanta, M. Wiessler, A. Jäschke, Bioconjugate Chem. 2012 23, 1382-1386; c) A. Niederwieser, A.-K. Späte, L. D. Nguyen, C. Jüngst, W. Reutter, V. Wittmann, Angew. Chem. Int. Ed. 2013, 52, 4265-4268; Angew. Chem. 2013, 125, 4359-4363; d) D. N. Kamber, L. A. Nazarova, Y. Liang, S. A. Lopez, D. M. Patterson, H.-W. Shih, K. N. Houk, J. A. Prescher, J. Am. Chem. Soc. 2013, 135, 13680-13683; e) A.-K. Späte, H. Bußkamp, 
A. Niederwieser, V. F. Schart, A. Marx, V. Wittmann, Bioconjugate Chem. 2014, 25, 147-154.

[13] L. I. Willems, N. Li, B. I. Florea, M. Ruben, G. A. van der Marel, H. S. Overkleeft, Angew. Chem. Int. Ed. 2012, 51, 4431 -4434; Angew. Chem. 2012, 124, 4507-4510.

[14] D. M. Patterson, K. A. Jones, J. A. Prescher, Mol. BioSyst. 2014, 10, $1693-$ 1697.

[15] a) C. Y. Zamora, M. d'Alarcao, K. Kumar, Bioorg. Med. Chem. Lett. 2013 23, 3406-3410; b) C. M. Cole, J. Yang, J. Šečkutè, N. K. Devaraj, ChemBioChem 2013, 14, 205-208.

[16] a) S. Hara, M. Yamaguchi, Y. Takemori, K. Furuhata, H. Ogura, M. Nakamura, Anal. Biochem. 1989, 179, 162-166; b) S. Hara, Y. Takemori, M. Yamaguchi, M. Nakamura, Y. Ohkura, Anal. Biochem. 1987, 164, 138-145.

[17] A.-K. Späte, J. E. G. A. Dold, E. Batroff, V. F. Schart, D. E. Wieland, O. R. Baudendistel, V. Wittmann, ChemBioChem 2016, 17, 1374-1383.

[18] Y.-J. Lee, Y. Kurra, W. R. Liu, ChemBioChem 2016, 17, 456-461.

[19] a) P. An, Z. Yu, Q. Lin, Chem. Commun. 2013, 49, 9920-9922; b) Z. Yu, T. Y. Ohulchanskyy, P. An, P. N. Prasad, Q. Lin, J. Am. Chem. Soc. 2013, $135,16766-16769$.
[20] a) S. Ito, Y. Tanaka, A. Kakehi, Bull. Chem. Soc. Jpn. 1976, 49, 762-766; b) S. Ito, Y. Tanaka, A. Kakehi, K. Kondo, Bull. Chem. Soc. Jpn. 1976, 49, $1920-1923$.

[21] a) W. Song, Y. Wang, Z. Yu, C. I. Rivera Vera, J. Qu, Q. Lin, ACS Chem. Biol. 2010, 5, 875-885; b) Y. Wang, W. Song, W. J. Hu, Q. Lin, Angew. Chem. Int. Ed. 2009, 48, 5330-5333; Angew. Chem. 2009, 121, 5434-5437.

[22] X. S. Wang, Y.-J. Lee, W. R. Liu, Chem. Commun. 2014, 50, 3176-3179.

[23] Y. Kurra, K. A. Odoi, Y.-J. Lee, Y. Yang, T. Lu, S. E. Wheeler, J. TorresKolbus, A. Deiters, W. R. Liu, Bioconjugate Chem. 2014, 25, 1730-1738.

[24] Z. Yu, Q. Lin, J. Am. Chem. Soc. 2014, 136, 4153-4156.

[25] Tetrazole-biotin 11 could not be applied owing to problems with its solubility.

[26] Z. Li, L. Qian, L. Li, J. C. Bernhammer, H. V. Huynh, J.-S. Lee, S. Q. Yao, Angew. Chem. Int. Ed. 2016, 55, 2002-2006; Angew. Chem. 2016, 128, $2042-2046$. 